\title{
Conceptualización del rol de la orientación al mercado en comercializadoras de químicos en la ciudad de Medellín
}

\author{
Conceptualization of the role of market orientation in distributors of chemicals in the \\ city of Medellín
}

\author{
Juan Santiago Calle Piedrahita ${ }^{1,2 *}$ \\ Iván Alonso Montoya Restrepo ${ }^{2}$ \\ Recibido 15 de junio de 2017, aceptado 4 de marzo de 2018 \\ Received: June 15, 2017 Accepted: March 4, 2018
}

\begin{abstract}
RESUMEN
Este artículo tiene como objetivo comprender el rol de la orientación al mercado que poseen las pequeñas, medianas y grandes comercializadoras de químicos en la ciudad de Medellín. Esta investigación es de enfoque cuantitativo, apoyada en el cuestionario de orientación al mercado, denominado MARKOR, con una escala Likert y compuesta por seis variables (orientación al mercado, orientación a la competencia, orientación interna, generación de información, análisis de información externa y capacidad de acción). Se aplicaron 343 encuestas a personal directivo durante los años 2015 y 2016. Los resultados obtenidos demuestran que las organizaciones aun con orientación al mercado no siempre generan ventaja competitiva. Las grandes comercializadoras tienen orientación al mercado con una valoración de 3,92/5,0, pero con preocupación por el análisis de la información externa, capacidad de acción y falta de orientación interna; las medianas empresas las clasifican con 3,89/5,0, pero con el peligro de no tener orientación a la competencia; y las pequeñas organizaciones las califican con 3,44/5.0, pero con deficiencia hacia la orientación interna.
\end{abstract}

Palabras clave: Orientación al mercado, mercadeo, estrategias, ventaja competitiva, capacidades de mercadeo.

\begin{abstract}
This article aims to understand the role of the market orientation of the small, medium and big chemical distributors in the city of Medellin. This research is a quantitative approach supported by the market orientation questionnaire called MARKOR with a Likert scale and composed of six variables (market orientation, competition orientation, internal orientation, information generation and external information analysis and action capacity). A total of 343 surveys were administered to managers during the years 2015 and 2016. The results show that even market-oriented organizations do not always generate competitive advantage. The large distributors have market orientation with a valuation of 3.92/5.0 but with respect to the analysis of the external information, capacity of action and lack of internal orientation; the medium-sized companies classify it with 3.89/5.0 but with the danger of not having an orientation to the competition; and small organizations rate it as 3.44/5.0, but deficient toward internal orientation.
\end{abstract}

Keywords: Market orientation, marketing, strategy, competitive advantage, marketing capabilities.

1 Facultad de Minas. Universidad Nacional de Colombia Escuela Nacional de Minas. Calle 80\#65-223, Medellín. Antioquia, Colombia. E-mail: jscallep@unal.edu.co

2 Facultad de Ciencias Agrarias. Universidad Nacional de Colombia. Calle 59ª \#63-20, Medellín. Antioquia, Colombia. E-mail: iamontoyar@unal.edu.co

Autor de correspondencia 


\section{INTRODUCCIÓN}

El mayor reto que poseen las organizaciones en la actualidad, es saber qué es lo que quieren los clientes, puesto que ellos son diferentes. Consecuentemente, las organizaciones que se enfocan en satisfacer en plenitud los gustos, deseos y necesidades de cada comprador, logran impulsar la recompra y la fidelización para otorgar mayor valor a la empresa. De igual manera, permite conocer detalladamente al público, basado en inteligencia e investigación de mercado. Es decir, las organizaciones se apoyan en conocer los clientes para ofrecer los productos que los satisfagan; sin embargo, para las comercializadoras de químicos, aunque logran satisfacer, en cierta forma, los requerimientos de los clientes, no es fácil ofrecer productos poco diferenciados en el mercado. Consecuentemente, la gestión se basa en análisis de ventas que llevan a olvidar un enfoque hacia al mercadeo, lo cual podría apoyar una mejora en la creación de valor.

La gestión de la organización está en constante cambio según los sucesos de la historia (Taylor se enfocó en proponer la teoría científica; Fayol se centró en la teoría de las funciones; Webber configuró la teoría de la burocracia; Elton Mayo se enfocó en las relaciones humanas, y así sucesivamente); es decir, según el período de la historia se aporta una nueva teoría que impulsa a la gestión de las organizaciones. Por consiguiente, deben ser flexibles para ajustar las estrategias al entorno y a los términos que impacten favorablemente la estructura de la organización; para ello, la orientación al mercado permite comprender mejor la función del mercadeo [1] en las organizaciones.

Así las cosas, la orientación al mercado presume la presencia de un conjunto de valores y de actitudes compartidas en la organización, para crear y configurar la creación de valor superior a los clientes [2-3]. Además, se obtiene una visión externa, donde se involucra el cliente, el competidor y los factores del entorno; asimismo, se analizan las condiciones internas, al incluir los stakeholders, los individuos y las funciones para desarrollar o proponer una respuesta competitiva en común, pero diferenciadora; incluso, promover la adopción de una visión estratégica, a largo plazo, según las capacidades de la empresa [4].

Hay estudios de resultados de la empresa encaminados a la reducción de la brecha entre mercadeo y orientación al mercado [5], configurado desde el aprendizaje vigilante del mercado, por medio de un sistema de advertencia anticipado [6]; es decir, gestionar las capacidades de comercialización, que lideran un papel importante para la implementación efectiva de la estrategia de mercadeo [7]; de modo similar, aportan a un mejoramiento [8] en el desarrollo estratégico de nuevos productos [9]. Otros estudios se encauzan a los resultados financieros y el mercadeo. Primero, la capacidad de comercialización tiene un mayor impacto en el rendimiento de la empresa, que las capacidades de investigación, desarrollo y operaciones [10]; segundo, la orientación al mercado aporta en el desempeño financiero y operacional, siempre y cuando sea moderado por la capacidad de I + D, y comercialización de la empresa [11]; tercero, las capacidades de marketing de orden superior son más importantes que las capacidades operativas, para explicar un rendimiento competitivo superior [12]; cuarto, las capacidades de la cadena de suministro, gestionada por tecnología de información (TI), son específicas de la empresa y difíciles de copiar en todas las organizaciones, para comprender la orientación al mercado [13] quinto, la flexibilidad estratégica se complementa con la eficacia, para ayudar a las empresas a gestionar condiciones del entorno después de una crisis [14], y adaptase al mercado; por último, pocos estudios se relacionan con la correspondencia de los negocios Business to Business (B2B) y la orientación al mercado; por ejemplo, las estrategias de innovación, tanto técnicas como no técnicas, conducen a un comportamiento de consumo sostenible [15], para aumentar la rentabilidad en creencia con la percepción del consumidor.

Incluso, un estudio de 367 PYMES australianas revela que se tienen dos claves para aumentar las capacidades de orientación al mercado: marca e innovación [16]; al mismo tiempo, la investigación de O'Cass y Ngo, descubrieron que simplemente tener orientación al mercado no es suficiente para crear valor y obtener ventajas en el mercado [17]; pero, las capacidades de mercadeo, a nivel de empresa, están asociadas con productos superiores basadas en la orientación al mercado [18]; en contradicción, Ballantyne y Aitken, expresan que la experiencia del servicio se vuelve fundamental para desarrollar y mantener la vida de una marca [19], y para crear nuevo valor percibido; paralelamente, Weill y Vitale, expusieron que la información en línea es importante para satisfacer a los clientes [20] al tener los datos al día. 
Otros estudios se enfocan a la incorporación de innovación y tecnología en los procesos de orientación al mercado; es decir, las relaciones entre las capacidades de comercialización, estrategias sostenibles basadas en la innovación y el comportamiento de consumo sostenible [15] aportan para obtener una visión del mercado internacional; asimismo, las empresas aprovechan una combinación distintiva de orientaciones y estrategias para tener éxito en diversos mercados internacionales [21]; por su parte, el estudio de Feng, Morgan y Rego, propone, según un panel de 612 firmas públicas de Estados Unidos de Norteamérica, que las capacidades de I $+\mathrm{D}$ de las empresas influyen de forma positiva en los efectos de las capacidades de comercialización en el crecimiento de la empresa, y que dichos efectos varían según las diferentes condiciones de los compradores [22], desde una orientación al mercado con enfoque estratégico teniendo en cuenta la dirección con productos afines. Lo anterior, visto desde una óptica internacional; sin embargo, desde una visión más específica, las empresas colombianas se han enfocado hacia la orientación a las ventas [23], y con poco ajuste a la orientación al mercado como enfoque estratégico.

Ahora bien, las empresas comercializadoras de químicos de la ciudad de Medellín, ofrecen similares productos genéricos para las diferentes industrias. Esto ha generado el desplome en las ventas y en la rentabilidad, por competir en función de precio de venta y con poco valor agregado en los productos o servicios similares durante las crisis económicas; lo cual, impulsó la necesidad de establecer nuevas leyes para evitar cierres empresariales; por ejemplo: crisis de 1997, se creó la ley de endeudamiento; crisis de 1999, se propone la ley de intervención económica; crisis de 2006, se debate la ley de acuerdo de reestructuración económica; crisis de 2012, se impone la ley de procesos de endeudamiento; y la crisis de 2015 se crea la ley de insolencia. Las nuevas leyes evitan quebrar y cerrar las empresas, porque se tiene una visión de ventas y no de mercadeo. También, se observa la visión cortoplacista de los gerentes, al no realizar investigaciones de mercado ni proyectar una gestión de marca [24], que logre posicionar la empresa en el medio.

En síntesis, el propósito de este artículo es comprender el rol de la orientación al mercado que poseen las pequeñas, medianas y grandes comercializadoras de químicos en la ciudad de Medellín, puesto que aún hay un imaginario de ventas asociado a la calidad, entrega, asesoría, atributos de los productos y relación beneficio/costo, y no hacia la orientación al mercado.

\section{ORIENTACIÓN AL MERCADO}

A continuación, se otorga una explicación de las definiciones del término orientación al mercado, y las herramientas utilizadas para comprender mejor los cambios que esta ha enfrentado durante estos últimos años.

La primera propuesta de orientación al mercado dada a conocer fue por Shapiro, quien la definió como un proceso de distribución de información del mercado al interior de toda la organización [25]; y la toma de decisiones es la sumatoria de lo operativo y estratégico.

Webster, enfocó la orientación al mercado hacia el cumplimiento por parte de la dirección, para: apoyar al comprador, analizar la planeación estratégica enfocada hacia este, preestablecer políticas de mercadeo, promover una visión hacia el cliente en toda la organización y medir la rentabilidad en función del mercadeo [26] y no en el producto.

Seguido, la escala de medición del grado de orientación al mercado de Naver y Slater, denominada MKTOR, consta de 15 ítems combinados con 9 anexiones, los cuales permiten medir tres componentes de la orientación al mercado con los resultados de la empresa: cliente, competencia e interdependencia funcional [3] [27].

La escala de Ruekert, no tiene un enfoque filosófico, es decir conocimiento abstracto o teórico del mercado, y representa las actuaciones de la organización, dirigidas al mercado; es una escala con una perspectiva operativa válida y fiable para canalizar el grado de adopción del mercado en las estructuras de las organizaciones [28]. Esta se configuró desde el constructo de Shapiro [25], Narver y Slater [27] y Jaworski, Kohli y Sahay [29], y está compuesta por 22 ítems agrupados en tres categorías: 1. orientación y uso de información sobre el cliente, 2. desarrollo de la estrategia de orientación al mercado, y 3. puesta en práctica de la estrategia de orientación al mercado. Aun así, se enfoca en una medición más cultural que operativa, principalmente hacia al cliente, y se olvida de la competencia y del entorno [28]. 
Kohli, Jaworski y Kumar, disponen de la escala de orientación al mercado, nombrada MARKOR [30]; una escala con 20 ítems que aporta a la medición del grado de orientación al mercado de las organizaciones, desde seis perspectivas: 1. orientación al mercado, 2. orientación a la competencia, 3. orientación interna, 4. generación de información, 5. análisis de la información externa, y 6. capacidad de acción. A su vez, se basa en la generación de inteligencia del mercado (entender el mercado y aspectos culturales), diseminación interna (analizar factores y aspectos operativos), y el desarrollo práctico de una acción dirigida al mercado (estrategias). En síntesis, otorga una visión general de los sucesos del mercado para incorporarlos en la organización y plantear estrategias.

De otro lado, la escala de Diamantopoulos y Hart se enfoca en identificar dimensiones subyacentes de la filosofía de la empresa y del concepto de mercadeo; en consecuencia, se sitúa en: propósito del mercadeo, función del mercadeo y orientación al cliente, pero se aleja del estudio del contexto de la competencia y el entorno [31].

Deshpandé, Farley y Webster propusieron la escala enfocada en analizar la cultura hacia el cliente desde 9 ítems, con tres categorías: orientación al competidor, aspectos culturales y operativos. Sin embargo, no incorporan el enfoque del entorno ni las políticas estratégicas de la organización [32].

La escala de Pelham de orientación al mercado involucra: el producto, la satisfacción del cliente y la orientación al competidor [33]; pero no valoriza la orientación al entorno externo; además, no hay factores subyacentes de la información y no define las políticas a largo plazo; es decir, tiene una visión operativa mas no cultural [34].

La escala de Deng y Dart, por su parte, se enfoca en cuatro categorías con 25 ítems: 1. orientación al cliente, 2. orientación a la competencia, 3 . coordinación interfuncional, y 4. énfasis en el beneficio [35]. Es decir, tiene un rumbo cultural más que operativo, y no analiza las condiciones del entorno.

La escala de Rivera Camino se apoya en una visión filosófica de orientación al mercado, al incorporar: cliente, comercializadores, competencia y macroentorno; analizados en tres dimensiones: dimensión de análisis, dimensión de coordinación y dimensión de acción [36].

La escala de Bisp, Harmsen y Grunert incorpora al cliente final, compradores, competidores y funciones de mercadeo (ventas, mercadeo, producción y direccionamiento estratégico). Además, consta de cinco dimensiones: inteligencia del mercado (actitud hacia el comprador, comportamiento hacia el comprador, actitud hacia el consumidor, comportamiento hacia el consumidor, actitud hacia los competidores, comportamiento hacia los competidores); nivel de actuación (actitud hacia las actuaciones operativas, comportamientos vinculados a actuaciones operativas, actitud hacia las actuaciones estratégicas, comportamiento vinculados a actuaciones estratégicas); capacidades (actitud hacia la velocidad de reacción, comportamiento vinculados a la velocidad de reacción, actitud hacia la puesta en práctica de los planes, comportamientos vinculados a la puesta en práctica de los planes); organización (actitud hacia discusión sobre el desarrollo del mercado, comportamientos vinculados a la discusión sobre el desarrollo del mercado, actitud hacia la coordinación de actividades, comportamientos vinculados a la coordinación de actividades); y apelación a la información (actitud hacia la prestación de los datos y comportamiento vinculado a la prestación de los datos) [37].

La escala de Desphande y Farley está compuesta por 10 ítems, configurada desde un análisis factorial de 44 enfoques: 15 de Naver y Slater; 20 ítems de la escala propuesta por Kohli, Jaworski y Kumar; y 9 adicionales de su propia autoría [38].

La escala de Gray, Matear, Boshoff y Matheson consta de 20 ítems y 3 dimensiones: orientación al cliente, orientación al competidor y coordinación interfuncional [39]; es decir efectúa la combinación de la escala de MKTOR, MARKOR y la propuesta de Deng y Dar [35].

Finalmente, la escala de Becjer y Homburg es aportada desde 5 subsistemas: organización, información, planificación, control y talento humano; las cuales generan 60 ítems en 16 subdimensiones: Organización (Jerarquía, contacto con el cliente, proceso organizativo); 
Tabla 1. Definiciones y características de Orientación al Mercadeo.

\begin{tabular}{|c|c|}
\hline Autor & Definiciones y características de orientación al mercadeo \\
\hline Shapiro & Proceso de distribución de la información del mercado en toda la organización [25]. \\
\hline Webster & $\begin{array}{l}\text { Apoyo de las directivas, planeación estratégica enfocada al mercado, prioridad de estrategias de mercadeo, } \\
\text { orientación al cliente y rentabilidad desde criterios del mercado [26]. }\end{array}$ \\
\hline Jaworski, Kohli y Sahay & Propuesta de la escala MARKOR [29] \\
\hline Kohli, Jaworsky y Kumar & Propuesta de la escala MARKOR ampliada [31] \\
\hline Ruekert & Obtención de información de los clientes para crear estrategias [28] \\
\hline Desphandé, Farley y Webster & Es una cultura organizacional enfocada prioritariamente hacia los clientes [32] \\
\hline Avlonitis y Gounaris & Es una filosofía organizacional la cual dirige el comportamiento de la empresa [41]. \\
\hline Pelham & $\begin{array}{l}\text { Está compuesta por tres categorías: conocimiento de las necesidades del cliente, análisis de la satisfacción } \\
\text { y orientación a la competencia [33]. }\end{array}$ \\
\hline Day & $\begin{array}{l}\text { Las habilidades y competencias de la organización promueven comprender el entorno del mercado y } \\
\text { lograr la efectiva gestión [1]. }\end{array}$ \\
\hline Cadogan y Diamantopoulos & Es un enfoque organizacional intrafuncional (interior de la empresa) e interfuncional (exterior - mercado) [42] \\
\hline Hunt y Morgan & $\begin{array}{l}\text { Recolección sistemática de información de los clientes y competidores para comprender el mercado y } \\
\text { establecer las respectivas estrategias. [43] }\end{array}$ \\
\hline Lambin & $\begin{array}{l}\text { Gestión de los recursos para obtener y analizar información enfocados a establecer estrategias incluyendo } \\
\text { a las comercializadoras [44] }\end{array}$ \\
\hline Deshpandé y Farley (1996) & Conjunto de procesos y actividades interfuncionales para lograr la satisfacción completa del cliente [38]. \\
\hline Han, Kim, y Srivastava & La orientación al mercadeo es un componente distinguido para la gestión de la organización [45]. \\
\hline Oliver & $\begin{array}{l}\text { Se enfoca en la lealtad del cliente para mejorar el proceso de fidelización del consumidor desde la } \\
\text { perspectiva actitudinal y comportamental. [46] }\end{array}$ \\
\hline Yang y Peterson & Analiza los costos del valor percibido en la satisfacción del cliente al haber un cambio en la lealtad del cliente [47]. \\
\hline
\end{tabular}

sistema de información (generación de información, diseminación de la información, almacenaje de la información); sistema de planificación (definición de objetivos, supervisión del entorno, selección de alternativas); sistema de control (comparación objetivos - resultados y análisis de desviaciones); sistemas de recursos humanos (selección, formación evaluación, promoción y recompensa-incentivos) [40]. En la Tabla 1 se resumen las diferentes definiciones y características de orientación al mercado.

En la Tabla 2, se observan las características de las diferentes escalas de medición de orientación al mercado y el enfoque de igualdad, diferencia positiva (leve o alta), con una clasificación en relación con: cultura (medición de actitud o estratégica), conducta (medición operativa) y filosófica (conocimiento abstracto o teórico del mercado).

Tabla 2. Diferentes Enfoques de orientación al mercado.

\begin{tabular}{|l|l|l|l|l|}
\hline \multicolumn{1}{|c|}{ Enfoque } & Cultural/actitud & Conducta/Operativa & Filosófico & \multicolumn{1}{c|}{ Características } \\
\hline Shapiro [25] & & & $\mathrm{X}(=)$ & Proceso de distribución equitativo \\
\hline Naver y Slater [27] & $\mathrm{X}(=)$ & & & 15 ítems - tres dimensiones Mktor \\
\hline Ruekert [28] & & & $\mathrm{X}(=)$ & 23 ítems - tres dimensiones \\
\hline Diamantropoulos y Hart [31] & & & $\mathrm{X}(=)$ & 12 ítems - cuatro dimensiones \\
\hline Kohli, Jaworski y Kumar [30] & & & & Procesamiento de Información MARKOR. \\
\hline Deshpandé, Farley y Webster [32] & $\mathrm{X}(=)$ & & & 9 ítems - enfoque hacia el cliente \\
\hline Pelham & $\mathrm{X}(=)$ & $\mathrm{X}(-)$ & & 9 ítems - tres dimensiones \\
\hline Deng y Dart [35] & $\mathrm{X}(+)$ & & $\mathrm{X}(=)$ & 25 ítems - Cuatro dimensiones \\
\hline Rivera Camino [36] & & & $\mathrm{X}(=)$ & Expectativas \\
\hline Lambin [44] & & $\mathrm{X}(=)$ & & 10 ítems - meta análisis - proceso \\
\hline Deshpandé y Farley [38] & & $\mathrm{X}(+)$ & $\mathrm{X}(-)$ & 20 ítems - seis dimensiones \\
\hline Bisp, Harmsen y Grubert [37] & $\mathrm{X}(+)$ & $\mathrm{X}(-)$ & $\mathrm{X}(+)$ & 60 ítems - Cinco dimens dimensiones \\
\hline Gray, Matear, Boshoff y Matheson [39] & $\mathrm{X}(-)$ & $\mathrm{X}(-)$ & Incluye la lealtad \\
\hline Becker y Homburg [40] & $\mathrm{X}(-)$ & $\mathrm{X}(+)$ & $\mathrm{X}(+)$ & Incorpora valor percibido de la lealtad \\
\hline Oliver [46] & $\mathrm{X}(+)$ & & \\
\hline Yang y Peterson [47] & & & \\
\hline
\end{tabular}


Según lo anterior, se puede decir que el propósito de la orientación al mercado es la búsqueda de mejores resultados en las organizaciones [48]. Por tanto, la escala de medición de orientación del mercado de Jaworski y Kohli [30] y Narver y Slater [3], están enfocadas hacia el cliente, la integración y el beneficio. Pero, Oliver y Yang, y Peterson, proponen otros métodos, en los cuales integran el desempeño del mercadeo con la evaluación del cliente, y la fidelización de los costos ocultos; es decir, está compuesta por la satisfacción, lealtad y retención del cliente [46-47].

En pocas palabras, se entiende que la orientación al mercado se apoya en: orientación al competidor, coordinación interfuncional, comprensión del consumidor y orientación multifuncional a largo plazo para cubrir y satisfacer las necesidades de los clientes. Di Benedetto \& Kim demuestran cierta relación directa entre complacencia del cliente y la gestión de ventas, si se incorpora el Custom Relationship Management (CRM) y el Customer Value Management (CVM) [49].

Desde las investigaciones de Jaworski y Kohli se propone que las organizaciones se deben orientar hacia el mercado desde un proceso operativo y no ideológico [50]. Además, Santos-Vijande, SanzoPerez, Alvarez-Gonzalez y Vazquez-Casielles expresan que la orientación al mercado ayuda a explicar los efectos de las organizaciones en la forma de gerenciar [51]. Desde este punto de vista, Slater y Narver encauzan los principios de medir el grado de orientación al mercado desde una perspectiva hacia el cliente, los competidores y el entorno [27].

En síntesis, hay diversas combinaciones de escalas de medición de la orientación al mercado, para comprender mejor la situación del entorno y ajustar las decisiones estratégicas sobre la gestión de comercializar, distribuir y vender, para crear valores superiores.

\section{CAPACIDADES SUPERIORES}

Desarrollos teóricos recientes se han centrado en la idea de que las empresas con capacidades superiores en mercadeo, son mejores generadoras de información acerca de los deseos y necesidades; también, son mejores en el impulso de productos y servicios para satisfacer necesidades y deseos [6]. Las capacidades superiores y la recolección de información otorgan a la empresa la posibilidad de generar valor, que conlleva al actuar con anticipación a los hechos de la competencia e impactar la diferenciación. Este proceso de generación y aplicación de capacidades, apoya la entrega de valor superior al cliente y beneficia a la empresa al aumentar la plataforma para una ventaja competitiva [52-55] de recursos y capacidades. De la misma forma, se instaura que la orientación al mercado se conforma por la habilidad para comprender y compensar a los clientes. Más aun, expone el concepto de activos basados en el mercado [1]; es decir, recursos acumulados en el transcurso del tiempo que posee la empresa, y las capacidades son los materiales que unen los activos para generar ventajas competitivas.

Las capacidades de mercadeo aportan a la generación de información del mercado, logrando así mejorar y actualizar la gestión interna y externa de la organización [56]. Sin embargo, Davidson, Whitaker y Dickens encontraron que las organizaciones, con menos capacidad de mercadeo, tienden a reducir el riesgo de la operación productiva [57]. Kotabe, Srinivasan y Aulakh, revelaron que las empresas al gestionar la capacidad de mercadeo, promueven a los gerentes a enfocar e implementar métodos para controlar los recursos y así lograr obtener ventajas especiales ante los competidores [11].

En síntesis, las capacidades de mercadeo aportan para entender las habilidades de la empresa, si éstas analizan el comportamiento del mercado y definen con claridad los nichos [6] a los cuales puede atender.

Desde las investigaciones de Konhli y Jawoski se propone que las organizaciones se deben orientar hacia el mercado desde un enfoque operativo y no ideológico. Además, Cadogan, Hooley, Douglas y Greenley, y Santos-Vijande, Sanzo-Pérez, ÁlvarezGonzález y Vázquez-Casielles expresan que la orientación al mercado ayuda a explicar los efectos de las organizaciones en la forma de gerenciar [58] [51].

Las empresas, al considerar el entorno interno y externo, aportan al diagnóstico para establecer las estrategias a seguir; para ello, se apoyan en los recursos, los cuales se combinan de la mejor forma para generar capacidades de mercadeo. Al mismo tiempo, la empresa configura, desde su interior, las estrategias para ampliar el espectro del mercado y poder satisfacer al cliente. Ver Figura 1. 


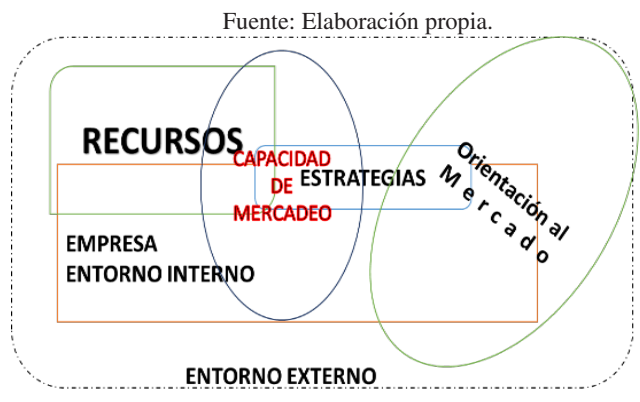

Figura 1. Configuración de capacidades de mercadeo, orientación al mercadeo y estrategia.

\section{METODOLOGÍA}

La investigación tiene un enfoque cuantitativo, aplicando el método deductivo de interpretación de resultados de la encuesta de orientación al mercado denominada MARKOR [30], compuesta por 34 ítems en seis variables (con escala Likert de uno a cinco, siendo cinco la más alta) (Ver Tabla 3), aplicada en forma presencial a gerentes y personal administrativo de comercializadoras de químicos en la ciudad de Medellín. De un total de 630 contactados solo 343 respondieron la encuesta. Este es un estudio cuantitativo, analizado por medio de la estadística descriptiva (media, desviación estándar y varianza).

La escala MARKOR utilizada en esta investigación es una adaptación de las dimensiones e ítems derivada de la original de Kohli, Jaworski y Kumar.

Tabla 3. Escala MARKOR compuesta por 34 ítems y seis variables.

\begin{tabular}{|c|c|}
\hline A & ORIENTACIÓN AL MERCADO \\
\hline 1 & Se analizan las características del cliente. \\
\hline 2 & $\begin{array}{l}\text { Las estrategias están orientadas para crear valor hacia los } \\
\text { clientes. }\end{array}$ \\
\hline 3 & $\begin{array}{l}\text { Las estrategias están enfocadas a obtener una ventaja } \\
\text { competitiva. }\end{array}$ \\
\hline 4 & $\begin{array}{l}\text { Los objetivos están orientados a la satisfacción de los } \\
\text { clientes }\end{array}$ \\
\hline 5 & $\begin{array}{l}\text { La satisfacción de los clientes se mide sistemática y } \\
\text { frecuentemente. }\end{array}$ \\
\hline 6 & Se presta atención al servicio de postventa. \\
\hline $\mathrm{B}$ & ORIENTACIÓN A LA COMPETENCIA \\
\hline 7 & $\begin{array}{l}\text { El personal de ventas comparte información con respecto } \\
\text { a las estrategias de los competidores. }\end{array}$ \\
\hline 8 & Se responde a las acciones de la competencia. \\
\hline 9 & $\begin{array}{l}\text { Se comunica o discute las fortalezas y estrategias de la } \\
\text { competencia desde la alta dirección. }\end{array}$ \\
\hline 10 & $\begin{array}{l}\text { Se contacta a los clientes en espera de una ventaja } \\
\text { competitiva. }\end{array}$ \\
\hline $\mathrm{C}$ & ORIENTACIÓN INTERNA \\
\hline 11 & $\begin{array}{l}\text { Los directivos de áreas funcionales visitan los clientes } \\
\text { actuales y potenciales. }\end{array}$ \\
\hline 12 & $\begin{array}{l}\text { Se comunican las experiencias (éxito o fracasos) con los } \\
\text { clientes. }\end{array}$ \\
\hline
\end{tabular}

\begin{tabular}{|c|c|}
\hline 13 & $\begin{array}{l}\text { Las áreas funcionales están integradas y coordinadas para } \\
\text { la satisfacción de los clientes en el nicho de mercado al } \\
\text { cual apuntan. }\end{array}$ \\
\hline 14 & $\begin{array}{l}\text { Los directivos comprenden las actividades de la empresa } \\
\text { en función de crear valor para el cliente. }\end{array}$ \\
\hline 15 & $\begin{array}{l}\text { Se comparten capacidades con otras unidades de la } \\
\text { organización. }\end{array}$ \\
\hline $\mathrm{D}$ & GENERACIÓN DE INFORMACIÓN \\
\hline 16 & $\begin{array}{l}\text { Se realiza investigación a los clientes para saber qué tipo } \\
\text { de servicio o productos ofrecer. }\end{array}$ \\
\hline 17 & $\begin{array}{l}\text { Con información obtenida en la investigación de mercados } \\
\text { se toman las decisiones. }\end{array}$ \\
\hline 18 & Detecta los cambios de la competencia fácilmente. \\
\hline 19 & $\begin{array}{l}\text { Contactan constantemente a los clientes para analizar } \\
\text { percepción de los servicios. }\end{array}$ \\
\hline 20 & Hacen estudios de cambios en la industria. \\
\hline 21 & Hay estudios de cambios en el entorno y sus efectos. \\
\hline $\mathrm{E}$ & ANÁLISIS DE LA INFORMACIÓN EXTERNA \\
\hline 22 & $\begin{array}{l}\text { Hay reuniones con gremios y otros para obtener } \\
\text { información de tendencias. }\end{array}$ \\
\hline 23 & $\begin{array}{l}\text { El personal de marketing dedica tiempo a analizar el } \\
\text { entorno y la competencia. }\end{array}$ \\
\hline 24 & $\begin{array}{l}\text { Se da a conocer lo bueno o lo malo de los clientes en la } \\
\text { empresa. }\end{array}$ \\
\hline 25 & $\begin{array}{l}\text { Se analizan datos sobre la satisfacción de los clientes ante } \\
\text { toda la organización. }\end{array}$ \\
\hline 26 & $\begin{array}{l}\text { Se informa oportunamente los cambios del entorno desde } \\
\text { la competencia. }\end{array}$ \\
\hline $\mathrm{F}$ & CAPACIDAD DE ACCIÓN \\
\hline 27 & $\begin{array}{l}\text { Se comunican los datos en función de la información para } \\
\text { reaccionar ante los cambios de la competencia. }\end{array}$ \\
\hline 28 & $\begin{array}{l}\text { Se admiten sugerencias, quejas y reclamos para mejorar el } \\
\text { servicio y/o productos. }\end{array}$ \\
\hline 29 & $\begin{array}{l}\text { Se revisan los procesos para generar nuevos productos o } \\
\text { servicios para los clientes. }\end{array}$ \\
\hline 30 & $\begin{array}{l}\text { Se realizan reuniones desde las área funcionales para } \\
\text { planear las respuesta a los clientes. }\end{array}$ \\
\hline 31 & $\begin{array}{l}\text { Competidor realiza campañas la empresa reacciona } \\
\text { inmediatamente. }\end{array}$ \\
\hline 32 & Hay coordinación entre las áreas funcionales. \\
\hline 33 & Se otorgan soluciones a las quejas reclamos y sugerencias. \\
\hline 34 & $\begin{array}{l}\text { Se adaptan a los cambios de productos sugeridos por los } \\
\text { clientes. }\end{array}$ \\
\hline
\end{tabular}

Se configuró una estadística de fiabilidad por medio del Alfa de Cronbach de 0,840 (Ver Tabla 4) y analizada a través del SPSS22.

Tabla 4. Estadística de Fiabilidad.

\begin{tabular}{|c|c|}
\hline Alfa de Cronbach & N de elementos \\
\hline, 840 & 343 \\
\hline \multicolumn{2}{|c|}{ Fuente: Elaboración propia a partir del SPSS22. }
\end{tabular}

Lo cual indica que está dentro del rango de excelente fiabilidad por estar por encima de 0,700 [64].

Ahora, analizando las diferentes escalas de medición de orientación al mercado, se opta por la escala de MARKOR, por estar más centrada en un enfoque de conducta operativa; es decir, ventas apoyadas por el mercadeo; en contraste con un enfoque de cultural (actitud hacia el mercadeo) y filosófico (debate epistemológico del término). Ver Tabla 2. 


\section{DISCUSIÓN}

Los siguientes son los hallazgos obtenidos de las encuestas a los gerentes y directivos de las empresas comercializadoras de químicos en la ciudad de Medellín (ver Tabla 5): El 88,24\% calificó con un 4.0/5.0, según la escala Likert, el análisis de las características de los clientes; un 2,94\% lo calificó con un 3.0; y solo el 8,82\% lo considera con un 5,0.

Del 88,24\%, con orientación al mercado, solo el 20,58\% de los empresarios calificó con 4.0 la generación de valor, y el 67,64\% lo calificó con 3.0; del 20,58\%, el $11,76 \%$ tenía un enfoque hacia la ventaja competitiva con $3.0 ; 2,94 \%$ con 5.0; y 5,88\% calificó con 4.0.

Del 67,64\% que calificaron en 3.0, el 58,82\% manifestó que lograban una ventaja competitiva con 4.0; y solo el $8,82 \%$ manifestó tener una ventaja competitiva con 3.0.

Sobre la gestión por orientación al mercado de la grande y mediana empresa, esta se preocupa por la satisfacción del cliente y la atención al servicio posventa; sin embargo, esta última adiciona en la gestión el estudio de las características del cliente; pero la pequeña empresa se enfoca en lograr una ventaja competitiva sin estudiar mucho al cliente.

Desde la variable orientación a la competencia, la pequeña empresa se enfoca en realizar contactos constantes con los clientes, pero la grande adiciona informes de los vendedores sobre el actuar de la competencia; la mediana, en cierta forma, no se preocupa por la competencia, solo la observa como aliada para copiar las estrategias que hacen las grandes.

Desde la orientación interna, la pequeña y la mediana empresa se enfocan en unir las diferentes unidades estratégicas de negocios y la comunicación interna de la empresa; pero la mediana aporta a visitar a los clientes con los vendedores para obtener nuevos datos de la competencia, indirectamente; no obstante, la grande se preocupa por visitar clientes y comunicar los sucesos de los clientes ante reuniones de mercadeo, ventas y de producción.

Desde la variable de análisis de información externa, la pequeña se adapta más rápido a los sucesos del entorno; la mediana se caracteriza por realizar estudios de mercados con los vendedores, y adaptarse lo más pronto posible al entorno; sin embargo, la grande empresa realiza investigación a los clientes para saber qué tipo de servicio o productos ofrecer, y detecta cambios de la industria fácilmente, pero al interior es lenta para adaptar los procesos de mercadeo.

En la capacidad de acción, una variable poca estudiada, se detecta que la grande empresa se basa en admitir peticiones, quejas, reclamos, sugerencias, denuncias y felicitaciones (PQRSDF), para mejorar el servicio y/o productos; también, revisan los procesos para generar nuevos productos o servicios para los clientes; por su parte, la mediana empresa se enfoca en realizar reuniones desde las diferentes áreas funcionales, para planear las respuesta a los clientes, demorándola y generando malestar hacia el cliente, quien desea una pronta solución; mientras, la pequeña empresa se preocupa por comunicar los datos ante las directivas para reaccionar a los cambios de la competencia y coordinar las áreas funcionales de la empresa para dar respuesta al PQRSDF.

En síntesis, la competitividad organizacional se representa en la configuración de ventajas competitivas [59] por medio de los recursos, las capacidades [60] y el enfoque estratégico que posee ésta. A su vez, se enlazan con indicadores de gestión de mercadeo, innovación y calidad en el servicio al cliente, lo cual permite posicionarse mejor en el medio. La empresa por sí sola, no logra competitividad, hay factores externos que impulsan a la configuración de ésta.

Tabla 5. Comparativo de empresas con orientación al mercado.

\begin{tabular}{|l|r|r|r|r|r|r|}
\cline { 2 - 8 } \multicolumn{1}{c|}{} & \multicolumn{2}{c|}{ Grande empresa } & \multicolumn{2}{c|}{ Mediana empresa } & \multicolumn{2}{c|}{ Pequeña empresa } \\
\hline Variable/Estadística & Media & $\begin{array}{c}\text { Desviación } \\
\text { estándar }\end{array}$ & Media & $\begin{array}{c}\text { Desviación } \\
\text { estándar }\end{array}$ & Media & $\begin{array}{c}\text { Desviación } \\
\text { estándar }\end{array}$ \\
\hline Orientación al mercado & 3,9233 & 0,4756 & 3,8900 & 0,5434 & 3,4433 & 0,8981 \\
\hline Orientación a la competencia & 3,6250 & 0,4887 & 2,8750 & 0,5985 & 3,4175 & 0,8483 \\
\hline Orientación interna & 2,7660 & 0,5623 & 3,2980 & 0,7270 & 3,0320 & 0,7526 \\
\hline Generación de información & 3,6600 & 0,5231 & 3,2500 & 0,7661 & 3,3333 & 0,9821 \\
\hline Análisis de información externa & 3,0400 & 0,6376 & 3,1340 & 0,9320 & 3,1320 & 0,7771 \\
\hline Capacidad de acción & 2,9588 & 0,5308 & 3,2925 & 0,8776 & 3,2088 & 0,7949 \\
\hline
\end{tabular}


Analizando la varianza en prestar atención al servicio de postventa de la grande empresa, está un 0,5281, pero la mediana empresa tiene una varianza 0,000 en el análisis de las características de los clientes, y la pequeña con una varianza de 1,367 en la variable de prestación postventa, la cual demuestra que no se preocupan por este elemento. Es decir, las empresas se diferencian entre ellas por las capacidades y recursos que poseen; además, la empresa estructura la identidad basada en las capacidades y recursos, y los factores externos e internos que afectan la competitividad y el beneficio de la empresa [11].

La grande y la mediana empresa se preocupa por el personal de ventas para que compartan información con respecto a las estrategias de los competidores $\left(\mathrm{s}^{2}=0,0866 ; 0,000\right)$; sin embargo, la pequeña empresa tiene un $\mathrm{s}^{2}$ de 1,600 , lo cual quiere decir que no se preocupa por analizar las estrategias de la competencia y efectuar correctivos.

Adicionalmente, la pequeña empresa tiene un $s^{2}=0,267$; la mediana empresa de $s^{2}=0,267$; y la grande de $s^{2}=0,4329$, en relación con la variable orientación a la competencia; por tanto, los directivos comprenden las actividades de la empresa en función de crear valor para el cliente, al identificar las necesidades a cubrir en el menor tiempo.

Ahora, desde una orientación interna, la grande empresa tiene una $\mathrm{s}^{2}=0,4329$; la mediana y la pequeña poseen una $s^{2}=0,267$; por consiguiente, las directivas, en cierta forma, si se preocupan por dar valor percibido a la empresa. Según Vorhies, Orr y Bush, las capacidades de mercadeo es un proceso integrador y sistemático para utilizar los recursos disponibles y aprovechar las capacidades del mercado [61]. En síntesis, el enfocarse con la orientación al mercado implica demostrar la gestión de las capacidades internas y externas de la organización, para realizar una combinación óptima que permita el desarrollo de una ventaja competitiva.

En la Tabla 6 se observa que la grande empresa tiene orientación al mercado, pero falla en el análisis de la información externa, capacidad de acción y falta de compromiso hacia el interior de la organización.
La mediana empresa tiene orientación al mercado, pero tiene una baja mirada hacia la orientación a la competencia y en la generación de información al medio; es decir, se considera una administración conservadora (Ver Tabla 7), como lo confirma Parada, Vázquez y Castro, quienes expresan que el direccionamiento estratégico de la organización se da, al considerar los recursos necesarios para maximizar los medios de información [62] y tomar las decisiones acertadas según los datos analizados.

La pequeña empresa tiene orientación al mercado, pero se preocupa por analizar la competencia y busca generar información tanto interna como externa (Ver Tabla 8) para ajustar ciertas políticas empresariales a su beneficio.

Tabla 6. Semáforos de orientación al mercado de la grande empresa.

\begin{tabular}{|l|cr|}
\hline Gran Empresa & \multicolumn{2}{|c|}{$\overline{\bar{x}}$} \\
\hline ORIENTACIÓN AL MERCADO & & 3,92 \\
\hline GENERACIÓN DE INFORMACIÓN & & 3,66 \\
\hline ORIENTACIÓN A LA COMPETENCIA & & 3,63 \\
\hline ANÁLISIS DE LA INFORMACIÓN EXTERNA & & 3,04 \\
\hline CAPACIDAD DE ACCIÓN & & 2,96 \\
\hline ORIENTACIÓN INTERNA & & 2,76 \\
\hline
\end{tabular}

Fuente: Elaboración propia.

Tabla 7. Semáforos de orientación al mercado de la mediana empresa.

\begin{tabular}{|l|rr|}
\hline Mediana Empresa & \multicolumn{2}{|c|}{$\bar{x}$} \\
\hline ORIENTACIÓN AL MERCADO & & 3,89 \\
\hline ORIENTACIÓN INTERNA & & 3,30 \\
\hline CAPACIDAD DE ACCIÓN & & 3,29 \\
\hline GENERACIÓN DE INFORMACIÓN & & 3,25 \\
\hline ANÁLISIS DE LA INFORMACIÓN EXTERNA & & 3,13 \\
\hline ORIENTACIÓN A LA COMPETENCIA & & 2,88 \\
\hline
\end{tabular}

Fuente: Elaboración propia.

Tabla 8. Semáforos de orientación al mercado de la pequeña empresa.

\begin{tabular}{|l|rr|}
\hline Pequeña Empresa & \multicolumn{1}{|c|}{$\overline{\mathrm{x}}$} \\
\hline ORIENTACIÓN AL MERCADO & & 3,44 \\
\hline ORIENTACIÓN A LA COMPETENCIA & & 3,42 \\
\hline GENERACIÓN DE INFORMACIÓN & & 3,33 \\
\hline CAPACIDAD DE ACCIÓN & & 3,21 \\
\hline ANÁLISIS DE LA INFORMACIÓN EXTERNA & & 3,13 \\
\hline ORIENTACIÓN INTERNA & & 3,03 \\
\hline \multicolumn{2}{|c}{ Fuente: Elaboración propia. }
\end{tabular}

Precisamente, Baker y Hart expresaron que la pequeña empresa le da dificultad orientarse al mercado por desconocer los clientes, el segmento de mercado y la no identificación de oportunidades del mercado [63]. 


\section{CONCLUSIONES}

Se pueden definir orientación al mercado como un proceso sistemático de conjuntos de recursos acumulados para lograr el beneficio o rentabilidad dentro de un marco limitado del entorno interno y externo de la organización. Es decir, es la combinación de la sumatoria de recursos limitados, para lograr el máximo de ello, incorporándolos en las situaciones de mercado. Por consiguiente, se incrementa la competitividad de la empresa al resolver problemas de satisfacción de las necesidades, gustos y deseos de los clientes. En consecuencia, las empresas tienen un enfoque hacia el mercado al impulsar la creación de nuevas oportunidades en el mercado que estimulan los ingresos.

Las organizaciones, con enfoque hacia la orientación al mercado, logran identificar el perfil del consumidor para diseñar, producir y entregar el producto según las necesidades o requerimientos del cliente.

A su vez, los consumidores de hoy exigen cada vez más, conocen los beneficios de antemano, consideran la calidad intrínseca, buscan precios justos y analizan el tiempo de entrega oportuna. Más aun, la orientación al mercado se realiza desde el enfoque del cliente como si este fuese una unidad estratégica de negocios de la organización, y no desde la visión de los propietarios de las empresas.

La orientación al mercado supone que los clientes son quienes toman las decisiones, no la empresa que produce o comercializa el producto. En consecuencia, ésta se apoya en la recolección y difusión de información de los clientes en la organización. Sumado a esto, hay un intercambio de conocimiento que genera una experiencia personalizada hacia el cliente.

Según los datos recolectados, la gran empresa considera la orientación al mercado como un elemento diferenciador $(3,92)$, ligado a la generación de información $(3,66)$, y con una orientación a la competencia $(3,63)$; sin embargo, la mediana empresa solo se preocupa por una orientación al mercado $(3,89)$, no basada en la información interna (CRM) $(2,76)$ ni en la capacidad de acción ante la competencia $(2,96)$; aun así, es preocupante para la pequeña empresa que es baja la orientación al mercado $(3,44)$, por no analizar la información del entorno $(3,13)$ ni la orientación interna $(3,03)$.

Consecuentemente, la orientación al mercado se basa en: analizar los competidores, comprender el cliente interno y el externo [25]. Es decir, la orientación al mercado se apoya en la visualización de conocer al cliente por medio de investigaciones de mercado y definir un perfil adecuado de este para generar ventaja competitiva.

Las futuras investigaciones aportarían a resaltar la necesidad de comprender el término orientación al mercado y sus características. También, contribuye a la posibilidad de aplicar en otros sectores de la industria e incorporar otras variables en el estudio de orientación al mercado, como: logística inversa, impacto de la tecnología del internet en las comercializadoras, responsabilidad social empresarial, cultura organizacional y comportamiento empresarial.

Finalmente, este estudio proporciona información a investigadores y gerentes sobre cómo gestionar y controlar las estrategias de orientación al mercado para comprender los efectos en las decisiones en la comercialización de químicos.

\section{REFERENCIAS}

[1] G. Day. "The capabilities of market-driven organizations". Journal of Marketing. Vol. 58. No 4, pp. 37-52. October, 1994. ISSN 1936-2011. DOI: 10.2307/1251915.

[2] R. F. Hurley and G. T. M. Hult. "Innovation, Market Orientation, and Organizational Learning: An Integration and Empirical Examination". Journal of Marketing. Vol. 62 № 3, pp. 42-54 Julio, 1998. ISSN 19362011. DOI: $10.2307 / 1251742$.

[3] S. Slater and J. Narver. "Customer-led and market-oriented: Let's not confuse the two". Strategic Management Journal. Vol. $19 \mathrm{~N}^{\mathrm{o}} 10$. octubre, 1998. ISSN: 1097-0266. DOI: 10.1002/ (SICI)1097-0266(199810)19:10<1001:AIDSMJ996>3.0.CO;2-4

[4] L. Santos, R. Vásquez and L. Álvarez. "Orientación al mercado en la estrategia de innovación, su efecto en las empresas industriales". Economía Industrial. Vol. 334 $\mathrm{N}^{\circ} 4$, pp. 93-106. 2000. ISSN 0422-2784. 
[5] K. R. Tuli, A. K. Kohli and S. G. Bharadwaj. "Rethinking customer solutions: From product bundles to relational processes". Journal of Marketing. Vol. 71 No $^{\circ}$ 3, pp. 1-17. 2007. ISSN: 0022-2429.

[6] G.S. Day "Closing the Marketing Capabilities". Journal of Marketing. Vol. 75 Pp. 183-195. Julio, 2011. ISSN: 0022-2429.

[7] N. Morgan, C. Katsikeas and D. Vorhies. "Export marketing strategy implementation, export marketing capabilities, and export venture performance". Journal of the Academy of Marketing Science. Vol. $40 \mathrm{~N}^{\circ}$ 2, pp. 271289. Marzo, 2012. ISSN: 0092-0703.

[8] G. H. Lim, K. S. Lee and S. J. Tan. "Gray marketing as an alternative market penetration strategy for entrepreneurs: Conceptual model and case evidence". Journal of Business Venturing. Vol. 16 No 4, pp. 405-427. 2001.

[9] D. Leonard- Barto. "Core capabilities and core rigidities: A paradox in managing new product development". Strategic Management Journal. Vol. 13 No S1, pp. 111-125. 1992. ISSN: 1097-0266. DOI: 10.1002/smj.4250131009.

[10] A. Krasnikov and S. Jayachandran. "The relative impact of marketing, research-anddevelopment, and operations capabilities on firm performance". Journal of Marketing. Vol. 72 No 4, pp. 1-11. Julio, 2008. ISSN: 1936-2011.

[11] M. Kotabe, S. Srinivasan and P. Aulakh. "Multinationality and firm performance: The moderating role of $\mathrm{R} \& \mathrm{D}$ and marketing capabilities". Journal of International Business Studies. Vol. 33 No $^{\circ}$, pp. 79-97. 2002. ISSN: 1970-2013.

[12] G. Hooley, J. Fahy, T. Cox, J. Beracs, K. Fonfara and B. Snoj. "Marketing capabilities and firm performance: a hierarchical model". Journal of Market-Focused Marketing Management. Vol. 4 № 3, pp. 259-278. octubre, 1999. ISSN: 1382-3019.

[13] F. Wu, S. Yeniyurt, D. Kim and S. Cavusgil. "The impact of information technology on supply chain capabilities and firm performance: A resource-based view". Industrial Marketing Management. Vol. 35 No 4, pp. 493-504. Mayo, 2006. https://doi. org/10.1016/j.indmarman.2005.05.003

[14] R. Grewal and P. Tansuhaj. "Building organizational capabilities for managing economic crisis: The role of market orientation and strategic flexibility". Journal of Marketing. Vol. $65 \mathrm{~N}^{\circ}$ 2, pp. 67-80. Abril, 2001. ISSN: 1936-2011.

[15] B. J. Mariadoss, P. S. Tansuhaj and N. Mouri. "Marketing capabilities and innovation-based strategies for environmental sustainability: An exploratory investigation of B2B firms". Industrial Marketing Management. Vol. $40 \mathrm{~N}^{\mathrm{o}}$ 8, pp. 1305-1318. Noviembre, 2011. https:// doi.org/10.1016/j.indmarman.2011.10.006

[16] B. Merrilees, S. Rundle-Thiele and A. Lye. "Marketing capabilities: Antecedents and implications for B2B SME performance". Industrial Marketing Management. Vol. 40 No 3, pp. 368-375. Abril, 2011. https://doi. org/10.1016/j.indmarman.2010.08.005

[17] A. O'Cass and L. V. Ngo. "Creating superior customer value for B2B firms through supplier firm capabilities". Industrial Marketing Management. Vol. $41 \mathrm{~N}^{\circ} 1$, pp. 125-135. Enero, 2012. https://doi.org/10.1016/j. indmarman.2011.11.018

[18] N. A. Morgan and R. J. Slotegraaf. "Marketing capabilities for B2B firms". Handbook on Business to Business Marketing. Edward Elgar Publishers. Northampton, Massachusetts. ISBN 978-1-84980 142-3. 2012.

[19] D. Ballantyne and R. Aitken. "Branding in B2B markets: insights from the servicedominant logic of marketing". Journal of Business \& Industrial Marketing. Vol. 22 No 6, pp. 363-371. 2007. ISSN: 0885-8624.

[20] P. Weill and M. Vitale. "What IT infrastructure capabilities are needed to implement e-business models?" MIS Quarterly Executive. Vol. $1 \mathrm{~N}^{\mathrm{o}}$ 1, pp. 17-34. Marzo, 2002.

[21] G. Knight and S. Cavusgil. "Innovation, organizational capabilities, and the bornglobal firm". Journal of International Business Studies. Vol. 35 N $^{\circ}$ 2, pp. 124-141. Marzo, 2004. ISSN: 0047-2506.

[22] H. Feng, N. A. Morgan and L. L. Rego. "Firm capabilities and growth: the moderating role of market conditions". Journal of the Academy of Marketing Science. Vol. $45 \mathrm{~N}^{\mathrm{o}}$ 1, pp. 76-92. Enero, 2017. ISSN: 0092-0703.

[23] R.A. Cueva. "Marketing: enfoque América latina". McGraw-Hill, $1^{\text {ra }}$. pp. 6-10. México. ISBN: 9701026632. 2000.

[24] E. Jassir Ufre. "Neuroimágenes en la investigación de mercados". Pensamiento. 
\& Gestión. № 26, pp. 73-93. 2009. ISSN 1657-6276.

[25] B. P. Shapiro. "What the hell is market oriented?" Harvard Business Review. pp. 119-125. 1988. URL: https://hbr.org/1988/11/ what-the-hell-is-market-oriented

[26] F. E. Webster. "The rediscovery of the marketing concept". Business Horizons. Vol. $31 \mathrm{~N}^{\circ}$ 3, pp. 29-39. Mayo-junio, 1988. ISSN: 0007-6813.

[27] J. C. Narver and S. F. Slater. "The effect of a market orientation on business profitability". Journal of Marketing. Vol. $54 \mathrm{~N}^{\mathrm{o}}$ 4, pp. 20-35. octubre, 1990. DOI: $10.2307 / 1251757$. ISSN: 1936-2011.

[28] R. W. Ruekert. "Developing a market orientation: an organizational strategy perspective". International Journal of Research in Marketing. Vol. $9 \mathrm{~N}^{\circ} 3$, pp. 225-245. Agosto, 1992. ISSN: 0167-8116.

[29] B. Jaworski, A. K. Kohli and A. Sahay. "Market-driven versus driving markets". Journal of the Academy of Marketing Science. Vol. $28 \mathrm{~N}^{\circ}$ 1, pp. 45-54. Enero, 2000. ISSN: 0092-0703.

[30] A. K. Kohli, B. J. Jaworski and A. Kumar. "MARKOR: a measure of market orientation". Journal of Marketing Research. Vol. $30 \mathrm{~N}^{\circ}$ 4, pp. 467-477. Noviembre, 1993. DOI: 10.2307/3172691. ISSN: 1964-2011.

[31] A. Diamantopoulos and S. Hart. "Linking market orientation and company performance: preliminary evidence on Kohli and Jaworski's framework". Journal of Strategic Marketing. Vol. 1 No 2, pp. 93-121. 1993. URL: https:// doi.org/10.1080/09652549300000007

[32] R. Deshpandé, J. U. Farley and F. E. Webster Jr. "Corporate culture, customer orientation, and innovativeness in Japanese firms: a quadrad analysis". Journal of Marketing. Vol. $57 \mathrm{~N}^{\circ} 1$, pp. 23-37. Enero, 1993. DOI: 10.2307/1252055. ISSN: 1936-2011.

[33] A. M. Pelham. "Market orientation and other potential influences on performance in small and medium-sized manufacturing firms". Journal of Small Business Management. Vol. $38 \mathrm{~N}^{\circ} 1$, p. 48. 2000. ISSN: 1540-627X.

[34] B. Stiegler. "Para una nueva crítica de la economía política: Sobre la miseria simbólica y el complejo económico-político del consumo". Nueva Sociedad. No 262, pp. 1-7. Marzo-abril 2016. ISSN: 0251-3552.

[35] S. Deng and J. Dart. "Measuring market orientation: a multi--factor, multi--item approach". Journal of Marketing Management. Vol. $10 \mathrm{~N}^{\circ} 8$, pp. 725-742, 1994. ISSN: 2333-6080.

[36] J. Rivera-Camino. "Re-evaluating green marketing strategy: a stakeholder perspective". European Journal of Marketing. Vol. 41, No 11/12, pp. 1328-1358. 2007. ISSN: 0309-0566.

[37] S. Bisp, H. Harmsen, and K. G. Grunert. "Improving measurement of market orientation, an attitude activity based approach". 25th EMAC Conference. University of Economic Sciences. Budapest Humgría. 1996.

[38] R. Deshpandé and J. U. Farley. "Understanding market orientation: A prospectively designed meta-analysis of three market orientation scales". 1996.

[39] B. Gray, S. Matear, C. Boshoff, and P. Matheson. "Developing a better measure of market orientation". European Journal of Marketing. Vol. 32 № 9/10, pp. 884-903, 1998. ISSN: 0309-0566.

[40] J. Becker and C. Homburg. "Market-oriented management: a systems-based perspective". Journal of Market-Focused Management. Vol. $4 \mathrm{~N}^{\circ}$ 1, pp. 17-41. Junio, 1999. ISSN: 1382-3019.

[41] G. Avlonitis and S. Gounaris. "Marketing orientation and company performance: industrial vs. consumer goods companies". Industrial Marketing Management. Vol. 26 No 5, pp. 385-402. Septiembre, 1997. https:// doi.org/10.1016/S0019-8501(96)00121-6

[42] J. W. Cadogan and A. Diamantopoulos. "Narver and Slater, Kohli and Jaworski and the market orientation construct: integration and internationalization". Journal of Strategic Marketing. Vol. 3 № 1, pp. 41-60. 1995. https://doi.org/10.1080/09652549500000003

[43] S.D. Hunt and R. M. Morgan. "The comparative advantage theory of competition,", Journal of Marketing. Vol. 59 N $^{\circ}$ 2, pp. 1-15. Abril, 1995. DOI: $10.2307 / 1252069$. ISSN: 1936-2011.

[44] J.-J. Lambin. "Marketing estratégico". McGRaw Hill Interamericana de España. 
$3^{\text {ra }}$. Madrid, España. pp. 8-11. ISBN: 9788448116118. 1995.

[45] J. K. Han, N. Kim and R. K. Srivastava. "Market orientation and organizational performance: is innovation a missing link?". Journal of Marketing. Vol. $62 \mathrm{~N}^{\circ}$ 4, pp. 30-45. Octubre, 1998. DOI: $10.2307 / 1252285$. ISSN: 1936-2011.

[46] R. L. Oliver. "Whence consumer loyalty?". Journal of Marketing. Vol. 63 pp. 33-44. 1999. DOI: 10.2307/1252099. ISSN: 1936-2011.

[47] Z. Yang and R. T. Peterson. "Customer perceived value, satisfaction, and loyalty: The role of switching costs". Psychology \& Marketing. Vol. 21 N$^{\circ} 10$, pp. 799-822. Agosto, 2004. DOI: 10.1002/mar.20030. ISSN: 1520-6793.

[48] C. Flavián, J. Jiménez, and F. J.Lozano. “Algunas consideraciones sobre la relación entre orientación al mercado y rentabilidad". Investigación y Marketing. Vol. 64, Pp. 34-39. 1999.

[49] C. A. Di Benedetto and K. H. Kim. "Customer equity and value management of global brands: Bridging theory and practice from financial and marketing perspectives: Introduction to a Journal of Business Research Special Section". Journal of Business Research. Vol. 69 № 9, pp. 3721-3724. Septiembre, 2016. https://doi.org/10.1016/j.jbusres.2015.03.048

[50] B. J. Jaworski and A. K. Kohli. "Conducting field-based, discovery-oriented research: lessons from our market orientation research experience". AMS Review. Vol. 7 No 1 , pp. 41-129. Junio, 2017. DOI:10.1007/ s13162-017-0088-5.

[51] M. L. Santos-Vijande, M. J. Sanzo-Pérez, L. I. Álvarez-González and R. Vázquez-Casielles. "Organizational learning and market orientation: interface and effects on performance". Industrial of Marketing Management. Vol. $34 \mathrm{~N}^{\circ}$ 3, pp. 187-202. Abril, 2005. https://doi.org/10.1016/j. indmarman.2004.08.004

[52] S. G. Bharadwaj, P. R. Varadarajan and J. Fahy. "Sustainable competitive advantage in service industries: a conceptual model and research propositions". Journal of Marketing. Vol. 57 No 4, pp. 83-99. Octubre, 1993. DOI: 10.2307/1252221. 1936-2011.

[53] J. C. Narver, S. F. Slater and B. Tietje. "Creating a market orientation". Journal of Market-Focused Management. Vol. $2 \mathrm{~N}^{\circ}$.
3, pp. 241-255. Septiembre, 1998. ISSN: 1382-3019.

[54] M. Tuominen, K. Möller and A. Rajala. "Marketing capability: a nexus of learningbased resources and a prerequisite for market orientation". Annual Conference of the European Marketing Academy. Mayo 1997.

[55] R. B. Woodruff. "Customer value: the next source for competitive advantage". Journal of the Academy of Marketing Science. Vol. $25 \mathrm{~N}^{\circ}$ 2, pp. 139-153. Marzo, 1997. ISSN: 0092-0703.

[56] S. S. Liu, X. Luo and Y.-Z. Shi. "Integrating customer orientation, corporate entrepreneurship, and learning orientation in organizations-in-transition: an empirical study". International Journal of Research in Marketing. Vol. 19 No 4, pp. 367-382. Diciembre, 2002. ISSN: 0167-8116.

[57] J. I. Davidson Jr, T. B. Whitaker and J. W. Dickens. "Grading, cleaning, storage, shelling, and marketing of peanuts in the United States". pp. 571-623. Diciembre 30, 2016. 1982 URL: https://apresinc.com/wp-content/ uploads/2015/12/PST-Chapter-15.pdf

[58] J.W. Cadogan, G. J. Hooley, S. P. Douglas, and G. Greenley. "Measuring Marketing Capabilities: A Cross-national Study". In Joint ANZMAC/EMAC symposium Marketing Networks in a Global Marketplace. Perth, Australia. Diciembre 1984.

[59] M. E. Porter. "Ventaja competitiva: creación y sostenimiento de un desempeño superior". Grupo Editorial Patria. $2^{\text {da }}$. pp. 25-54. Ciudad de México México. ISBN-10: 6077440809. Marzo, 2015.

[60] R. A. Cardona. "Estrategia basada en los recursos y capacidades. Criterios de evaluación y el proceso de desarrollo". In Forum Doctoral, 2013. o 4, pp. 113-147. Mayo-julio, 2013. ISSN: 2027-2146.

[61] D.W.Vorhies, L.M.OrrandV.D. Bush. 'Improving customer-focused marketing capabilities and firm financial performance via marketing exploration and exploitation". Journal of the Academy of Marketing Science. Vol. 39 № 5, pp. 736-756. octubre, 2011. ISSN: 0092-0703.

[62] M. Castro, D. Parada and V. González. "El marketing desde una visión empresarial basada en los recursos: una aproximación conceptual a las capacidades estratégicas de marketing y al desarrollo de sus instrumentos de medida". Investigaciones Europeas de 
Dirección y Economía de la Empresa. Vol. $1 \mathrm{~N}^{\circ} 1$, pp. 31-67. 2004. ISSN: 1135-2523

[63] M. Baker and S. Hart. "The marketing book". Routledge-Elsevier Ltd. ${ }^{\text {ta }}$. pp. 3-18. Gran Bretaña. ISBN: 978-0-7506-8566-5. 2008.
[64] R. Ledesma, G. Molina Ibañez and P. Valero Mora. "Internal consistency analysis by means of Cronbach's Alpha: a computer program based on dynamic graphics". Psico-USF. Vol. $7 \mathrm{~N}^{\circ} 2$, pp. 143-152. 2002. 